\title{
The Cost of Astronomy
}

\section{Publishing fees in astronomy: Is something rotten in the case of Denmark?}

\author{
Bertil F. Dorch ${ }^{\star}$ \\ ${ }^{1}$ University Library of Southern Denmark, SDU, Campusvej 55, DK-5230 Odense, Denmark
}

\begin{abstract}
Using Scopus and national sources, I have investigated the evolution of the cost of publishing in Danish astronomy on a fine scale over a number of years. I find that the number of publications per year from Danish astronomers increased by a factor of four during 15 years: naturally, the corresponding potential cost of publishing must have increased similarly. The actual realized cost of publishing in core journals are investigated for a high profile Danish astronomy research institutions. I argue that the situation is highly unstable if the current cost scenario continues, and I speculate that Danish astronomy is risking a scholarly communication collapse due to the combination of increasing subscription cost, increased research output, and increased direct publishing costs related to Open Access and other page charges.
\end{abstract}

\section{Introduction}

In March 2016, The New York Times [5] wrote that "Journal publishers collectively earned 10 billion USD last year, much of it from research libraries, which pay annual subscription fees ranging from 2,000 to 35,000 USD per title if they don't buy subscriptions of bundled titles, which cost millions. The largest companies, like Elsevier, Taylor \& Francis, Springer and Wiley, typically have profit margins of over 30 percent"

In the same article, Peter Suber, the Director of Harvard's Office of Scholarly Communication was quoted saying that "the prices have been rising twice as fast as the price of health care over the past 20 years, so there's a real scandal there to be exposed". One solution, Suber was quoted saying, "was to persuade researchers to publish in Open Access journals like those under the umbrella of the Public Library of Science,or PLOS, co-founded by Dr. Eisen at Berkeley. But that financial model requires authors to pay a processing charge that can run anywhere from 1,500 to 3,000 USD per article so the publisher can recoup its costs".

In 2014, The Welcome Trust [2] published details of how much the research foundation spent on Open Access publishing during 2012-2013 in an attempt to make the debate around the costs of open access publishing more evidence-based: The Welcome Trust spent approximately 3.9 million GBP on APCs for 2126 articles in the year 2012-2013. Overall, the average APC for publication in hybrid journals is 2,727 USD.

A study, that is in a way a supplementary to that of [2] was presented by Gray [6] to show that the APCs paid to publish Open Access content, and the indirect costs of handling Open Access mandates

\footnotetext{
^e-mail: bfd@bib.sdu.dk ORCID: 0000-0003-2594-6778
} 
Table 1. Core journals of Danish astronomy defined as journals with contributions from authors coming from Denmark during 2010-2014 according to Scopus (left column), and the average annual number of publications in the corresponding journals (right column).

\begin{tabular}{lr}
\hline Journal & Average annual number of publications \\
\hline Astronomy and Astrophysics & 55.5 \\
Astronomical Journal & 3.1 \\
Annales Geophysicae & 2.7 \\
Astrophysical Journal* & 57.3 \\
Astronomische Nachrichten & 3.8 \\
Experimental Astronomy & 1.1 \\
Proceedings of the International Astronomical Union & 6.6 \\
ICARUS journal & 1.1 \\
International Journal of Modern Physics A-D & 7.5 \\
Journal of Cosmology and Astroparticle Physics & 8.6 \\
Monthly Notices of the Royal Astronomical Society* & 29.7 \\
Publication of the Astronomical Society of Japan & 0.5 \\
Publications of the Astronomical Society of the Pacific & 1.1 \\
Physics of the Earth and Planetary Interiors & 1.3 \\
Planetary and Space Science & 1.8 \\
Solar Physics & 2.2 \\
Space Science Reviews & 1.1 \\
\hline
\end{tabular}

costs may be unexpectedly high for individual institutions: the study added an estimate for the other publication charges currently paid by research institutions, a significant element which has been neglected by recent studies. When page and color charges are included, the total cost to institutions as of 2013-2014 is around 18.5 percent over and above the cost of journal subscriptions-11 percent from APCs, 5.5 percent from indirect costs, and 2 percent from other publication charges. For the Great Britain as a whole, this represents a total cost of publication around 213 million GBP against a conservatively estimated journal spend of 180 million GBP, with non-Open Access APCs representing around 3.6 million GBP [6].

Recently, the Max Planck Digital Library White Paper on Open Access by [3] makes the case for a large-scale transformation of the current scholarly publishing system to an Open Access model. The White Paper advocates that there need to be "a shared understanding that the money currently locked in the journal subscription system must be withdrawn and re-purposed for open access publishing services".

Gauffriau et al. [4] conducted an analysis showing that the funds spent on journal licenses by universities in Denmark is sufficient to pay for the number of publications from the same universities should they be subjected to an APC. In accordance with the Max Planck Digital Library White Paper, the authors found that the average cost per publication of 3,600 Euro in Denmark is only a little lower than a similar calculation by [3] yielding a global level cost per publication of 3,800 Euro [4].

\section{Danish astronomy as a case}

To investigate the cost of scholarly publishing on an even finer scale - and to include all direct publication costs, as argued by e.g. [6], I used astronomy and astrophysics in Denmark as a case. It can be argued that astronomy in several ways is both a traditional field of natural science with roots back 
Table 2. Publishing fees associated with core journals of Danish astronomy (article charges in Euros, 2017).

\begin{tabular}{llrr}
\hline Journal & Publisher & APC (Euro) & Gold OA \\
\hline Astronomy and Astrophysics & EDP & - & 400 \\
Astronomical Journal & IOP & 763 & - \\
Astrophysical Journal & IOP & 763 & - \\
Journal of Cosmology and Astroparticle Physics & IOP & - & 1600 \\
Monthly Notices of the Royal Astronomical Society & Oxford & - & 2175 \\
Publications of the Astronomical Society of the Pacific & Chicago & 1000 & - \\
Solar Physics & Springer & - & 2200 \\
\hline
\end{tabular}

to the origin of the scientific method and beyond, but also a first-mover e.g. with respect to adopting Open Science practices including Open Access all the way back to the birth of the Internet. Thanks to arXiv.org, within the field of astronomy and astrophysics, Open Access has generally been achieved through Green Open Access self-archiving.

The core journals of Danish astronomy are defined here by considering all astronomy "documents" with contributions from authors coming from Denmark during 2010--2014 according to Scopus (46 journals in total), and selecting the set of journals with recurrent authorships (i.e. no one-hit wonders) resulting in the journals listed in Table 1.

During the period 2000-2014 the total number of publications per year increased from around a hundred articles to several hundred, cf. Fig. 1 (left). Mostly, the increase comes from publishing in the identified core journals, which also happen to be journals with related publishing fees, i.e. either Golden Open Access APCs or non-Open Access article charges, cf. Fig. 1 (right). Only a minor part of the articles are published in Platinum Open Access journals (journals without associated APCs or fees).

On the one hand, even though most core astronomy journals allow or even encourage selfarchiving, some of them do in fact offer a Golden Open Access option corresponding to an APC. On the other hand, some core astronomy journals require APCs to be paid that are not related to Open Access. An example of the latter is ApJ from The American Astronomical Society, which is published by IOP Publishing. Article charges are calculated by The American Astronomical Society by counting units of information in the digital form that the author supplies called "digital quanta", i.e. units of information in digital form that can include words, figures, tables, digital-only components etc.

This way of calculating article charges makes the cost of an article a unique value, as opposed to the typical Open Access APC which is fixed for a particular journal or publisher, cf. Table 2.

In order to get a handle on the typical non-Open Access article charge for a paper published in ApJ, I calculated the average cost of publishing in this journal by the DARK Cosmology Center at University of Copenhagen during 2012-2015. These four years, scholars at DARK together with international colleagues published a substantial number of articles in ApJ and were billed by IOP for a total of 32 of these articles costing DARK all in a sum of 181,980 DKK. The average cost per article per year ranged between 5,033 and 9,100 DKK corresponding to an overall average of 763 Euro.

As an example for comparison to DARK's 32 ApJ articles during four years, a total 1030 articles with authors from Denmark was published in The Astrophysical Journal during a quarter of a century, 1990-2016. Of these, 775 articles also had co-authors from the USA, 411 articles have co-authors from the UK, 327 have co-authors from Germany, 264 have co-authors from Italy, 206 have co-authors from France etc. (Scopus, March 2016). The average is thus almost 40 ApJ articles per year (nearly 160 papers per four years) corresponding to potentially tens of thousands of Euros per year. 

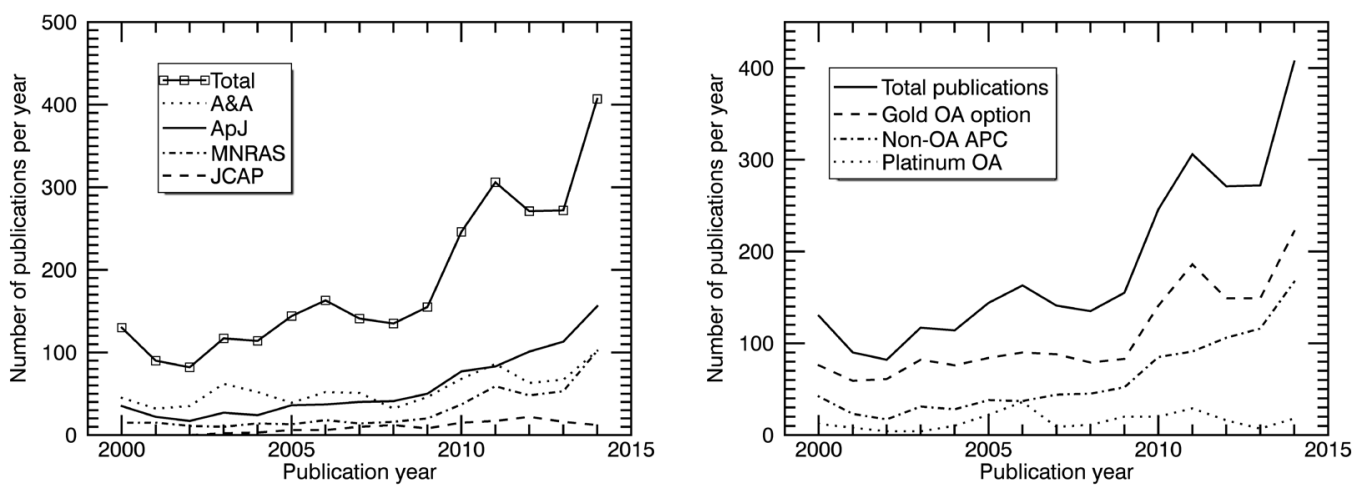

Figure 1. Left: The total number of publications per year from 2000 to 2014 (solid squared curve). Also shown are the number of publications per year in core journals A\&A; ApJ, MNRAS and JCAP. Right: The total number of publications per year broken down into publications with a Gold Open Access option, a non-Open Access article charge (APC) and Platinum Open Access (no APC's or fees).

\section{Discussion and outlook}

Non-Open Access or Toll Access publishing in astronomy and astrophysics is traditionally supplemented by Green Open Access through authors' self-archiving into the open repository arXiv.org at Cornell University Library. This method of achieving Open Access has been the modus operandi for astronomy and astrophysics even prior to the invention of the term Open Access around the millennium: arXiv.org has now been in operation for more than a quarter of a century.

The estimated (maximum) potential annual cost of APCs related either Golden Open Access publishing or traditional Toll Access publishing in astronomy and astrophysics — based on the publication record in Denmark during 15 years — are the same order of magnitude, cf. Fig. 2.

Consequently, the potential cost of publishing in astronomy and astrophysics in Denmark is on the order of a several 100,000 Euros per year, including both Open Access and non-Open Access publishing.

Publishing in astronomy is of course funded in several different ways, e.g. through international collaboration, and potential costs — future or present —- are not only realized in Denmark. However, it remains to be seen, if the current publishing system is sustainable in the Open Access era, and if the realization of increasing costs will quench the apparent growth of Danish astronomy as reflected by the publication output from 2000 and thereafter. Additionally, the important factor of subscription costs needs to be included when assessing the evolution of the said economy.

\section{Acknowledgements}

I wish to acknowledge the DARK Cosmology Center at University of Copenhagen for providing me with data regarding the center's publishing in ApJ. Furthermore, I would like to acknowledge SDU, The University of Southern Denmark, the organizing committee of the LISA 8 conference, and the International Astronomical Union for supporting LISA 8. 


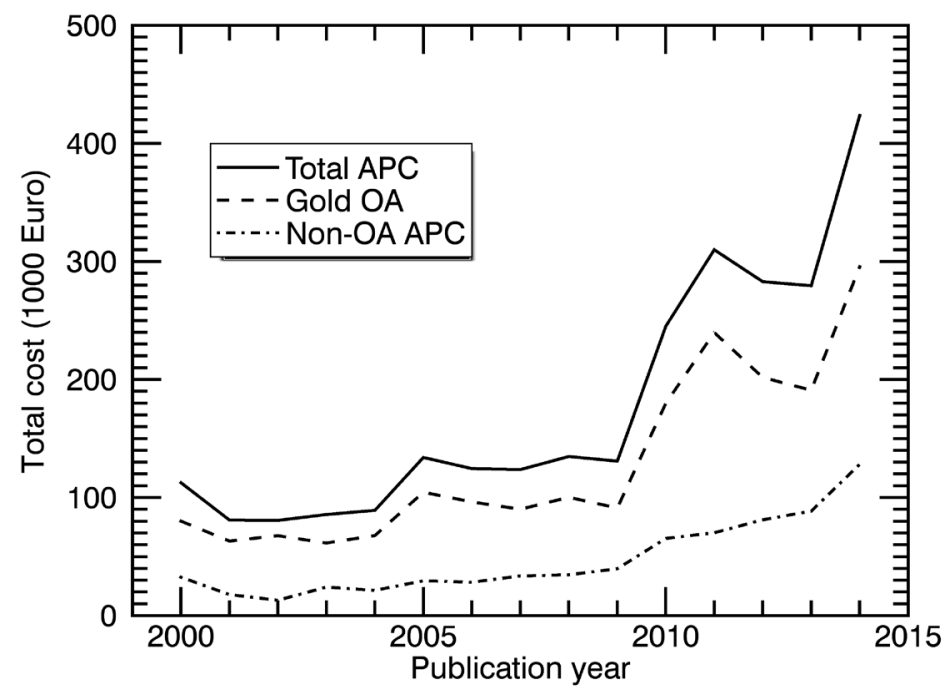

Figure 2. The potential (maximum) total cost of Danish astronomy publishing per year from 2000 to 2014 (solid curve). The potential cost per year is broken down into Gold Open Access fees (dashed curve) and non-Open Access article charges (dashed-dotted curve).

\section{References}

[1] Van Noorden, R., Nature 495, 426-429: doi:10.1038/495426a (2013)

[2] B.-C. Björk, D. Solomon, Developing an Effective Market for Open Access Article Processing Charges (Report from Wellcome Trust, United Kingdom, 2014)

[3] R. Schimmer, K. K. Geschuhn, A. Vogler, Disrupting the subscription journals' business model for the necessary large-scale transformation to open access (Max Planck Digital Library Open Access Policy White Paper, Germany, 2015)

[4] M. Gauffriau, A. V. Larsen, K. H. Lianez, Transition from the License to read model to Open Access publishing. A Danish study (DEFF Report, Denmark, 2016)

[5] K. Murphy, "Should all research papers be free?", The New York Times, March 12 (2016)

[6] A. Gray, Publications 3, 248-262 (2015) 
